\title{
Bats Sonar Calls and Its Application in Sonar Systems
}

\author{
Tadeusz Gudra ${ }^{1}$, Joanna Furmankiewicz ${ }^{2}$ and Krzysztof Herman ${ }^{1}$ \\ ${ }^{1}$ Wroclaw University of Technology \\ ${ }^{2}$ University of Wroclaw \\ Poland
}

\section{Introduction}

Sonar system operating in gas medium (air) are based on echolocation phenomenon (active sonar). Many scientist and specialists in the field of air coupled ultrasound localisation systems have been inspired by echolocation mechanism of bats. Those mammals mastered echolocation perfectly using ultrasound waves. A resolution of so called real time biosonar is out of range of human made equivalent due to scientific and technological limitations. The main aim of the chapter presented below is to introduce book readers with the echolocation mechanism, evolved in bats, which are an example of animals that have achieved perfection in biosonar usage. The next problem that authors cover is an evolution of sonar systems from simple one beam ranging echolocation devices to advanced multibeam array beamformers operating with digital signal as serviceable form of information representation. Some properties of ultrasound waves in gas medium and physical phenomena involved with air coupled ultrasound waves generation, transmission and detection are described in the chapter as follows: (1) generation of ultrasound wave in gas medium, (2) a short review of air operating transducers including piezoceramics, sandwich, electrostatic, EMFi and MEMS, (3) problem of ultrasound wave transmission in gas medium (the influence of temperature and static pressure on speed of sound, attenuation in air) and range equation, (4) reception and detection of ultrasound waves, target strength. Considering the fact that the detection of echo signal does not give us an information about target a review of signal processing methods is also presented. Taking into account global trend in applying digital signal processing methods to sonar application authors describe some of DSP solutions which are adaptation of echo signal processing by bats at higher level of their auditory system. In order to explain some important issues from bat echo analysis, authors to present some information about bat auditory system and the role of its particular organs and auditory pathways in spatial information processing.

\section{The bases of echo imaging in echolocating bats}

Echolocation plays the most important role in the environment perception in the echolocating bats and odontocete cetaceans. The sonar system of bats and dolphins evolved however in a different way, because of differences in animal size, environment, prey and sound propagation in the air and water $\mathrm{Au}$ (2004). Most of the over 1200 bat species echolocate. Echolocation is 
used for spatial orientation and prey pursuing. Bats ordinarily emit echolocation calls when moving in light and dark, suggesting that echolocation is a very important component of their sensorimotor system. Nonetheless, echolocation is not the only sensory system of bats, since they probably make use of all senses (including vision, olfaction and kinesthetic sense) to integrate them for cognitive processing Masters \& Harley (2004).

In the echolocating sensing system of bats, ultrasound vocalisation is actively propagated over the air and returning echoes are used for determination of the position, size and structure of sonar target. A bat can determine between its own echo and echoes coming from other individuals. Sometimes bats can even eavesdrop on emitted sound and its echo of conspecifics and other species to find good foraging sites and forage with members of social group Balcombe \& Fenton (1988), Dechmann et al. (2009). These findings and other researches show that echolocation pulses serve as acoustic cues in communication of bats (see review in Jones (2008)).

\subsection{Sound emission}

Sound emission in bats is produced in the larynx, a common sound generator in all mammals. Bats emit calls through the mouth or the nostrils. Nostril emission have advantage when foraging, because a bat can fly and echolocate with the prey in mouth. Moreover, the emission of sound through two nostrils results in interference of emitted sound beams. The interference pattern depends on the distance between nostrils. If it's half of the wavelength of the emitted frequency, the sound energy straight ahead will be intensified, and attenuated on either side, resulting in a narrowing of the sound beam. Experiments with a leaf-nose bat Carollia perspicillata (family Phyllostomidae) showed that when one of the two nostrils is blocked the extend of the sound beam doubles in the horizontal plane Fig. (1) Hartley \& Suthers (1987).

Bats using the nostrils often have complex noseleaves composed of fold of skin and cartilage. The noseleaves have varying complexity and shape: e.g. shape of horseshoes in horseshoe bats Rhinolohphidae or vertical blade-like noseleaf in leaf-nosed bats Phyllostomidae. Noseleaf acts as acoustic lens, focusing the sound into a narrow beam, which cause increasing of directionality of emitted calls and enhanced echolocation performance. The experiments with Carollia perspicillata indicated that if the noseleaf is removed, the vertical beam width increased from 60 to $120[\mathrm{deg}]$ Hartley \& Suthers (1987).

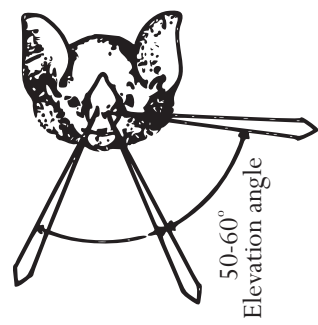

Horizontal angle $50-60^{\circ}$

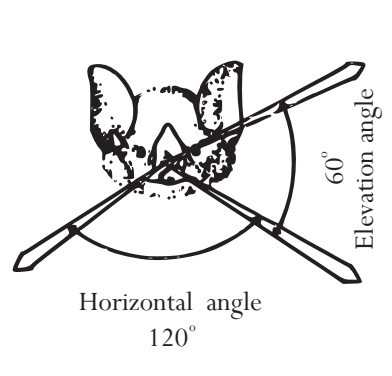

Horizontal angle $50-60^{\circ}$

Fig. 1. The relation between the width of the sound beam and nose-leaf and nostril emission in Carollia perspicillata (adapted from Hartley \& Suthers (1987)). 


\subsection{How bats avoid deafening?}

The intensity of the emitted echolocation pulses may be up to maximum level above 140 $[d B]$ of sound pressure level (SPL) Surlykke \& Kalko (2008). This is loud enough to cause hard pain and hardly damage the eardrum and cochlea. Bats posses special adaptations to avoid deafening. Echolocation calls are emitted in short bursts. During the burst, bats make themselves temporarily deaf. The deafening is caused by the contraction of the muscle stapedius, which is attached to stapes (a small ossicle in the middle ear, see the chapter 'Auditory adaptations to sound perception'). Just before a bat emits an echolocation pulse, the stapedius contracts and pulls the stapes away from the oval window Henson JR (1965). The sound cannot be passed to the cochlea, which prevent the damage of this delicate structure. In the final stage of pursuing for an insect, a bat's sonar pulse repetition rate may exceed 200 $[\mathrm{Hz}]$, and its stapedius muscle may operate at the same frequency - one of the highest rates recorded in vertebrate muscle Altringham (1999).

\subsection{The diversity of bat echolocation calls}

The diversity of echolocating bats and its habitat leads to diversity of acoustic signals used for echolocation and therefore acoustic information received from echoes. Bats' sonar pulses are usually described as narrowband constant frequency calls (CF) and broadband frequency modulated pulses (FM), however there are many combinations of those two call types Fig. (2) Neuweiler (2000). FM pulses starts at high frequency and sweeps downward to progressively lower frequency. They last for a short period of time, usually up to several ms. There is interspecies and between habitat variation in the slope of the pulse. CF signal is a pure tone or only slightly modulated in frequency at the beginning. In horseshoe bats (Rhinolophidae), hipposiderid bats (Hipposideridae) and mormoopid Pteronotus parnellii the CF tone ends and/or starts with short FM part. CF pulse usually last for $10-100$ [ms].

Many FM bats used open, semi-open, edges of forest, separating pulse from echo in the time and discriminating delay in echo when estimating target distance, whereas CF bats usually forage in clutter environment and emit long-duration calls separating pulse and echo in frequency Neuweiler (2000).

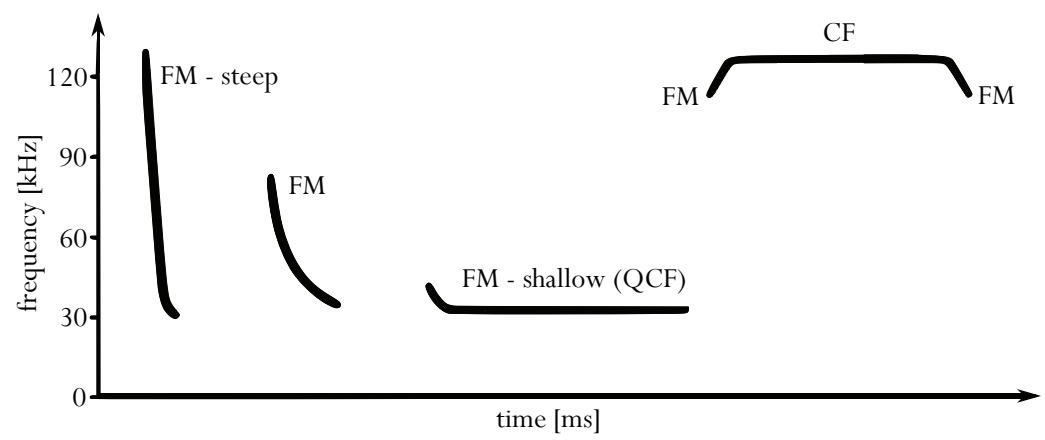

Fig. 2. The main categories of bat echolocation pulses: broadband FM sweeps, narrowband quasiconstant frequency pulse with short FM element (FM-QCF) and constant frequency (CF) call.

An echolocating bat emits pulses with irregular time intervals. During pauses between pulses no information is available. The time without information varied between species, habitats and pulse type. The ratio of the time during which a signal is present to the entire duration of 
the time from one call onset to the next is called duty cycle. FM bat echolocate on low-duty cycle, i.e. most of the echolocation sequence is occupied by interpulse interval (gaps between pulses), and the pulses covered usually less than $20[\%]$ of the sequence. CF bat produces high-duty cycle sequence, where signals occupied more than $50[\%]$ of the sequence time Jones (1999).

The echolocation pulse sequence of a flying bat foraging for insects consists usually of three phases. When a bat is searching for an insect it emits search pulses (search phase). As soon as the bat detect target, it begins to pursuit it, emitting signals faster, progressively decreasing intervals between them in order to avoid overlap between the outgoing signal and the returning echo. This is called approach phase and a bat can emit calls with the signal repetition rate up to $40-50 / \mathrm{s}$. Just before the bat captures an insect, it emits a final buzz, called also terminal buzz or feeding buzz Fig. (3). During the final buzz, the duty cycle increases to about $90[\%]$.
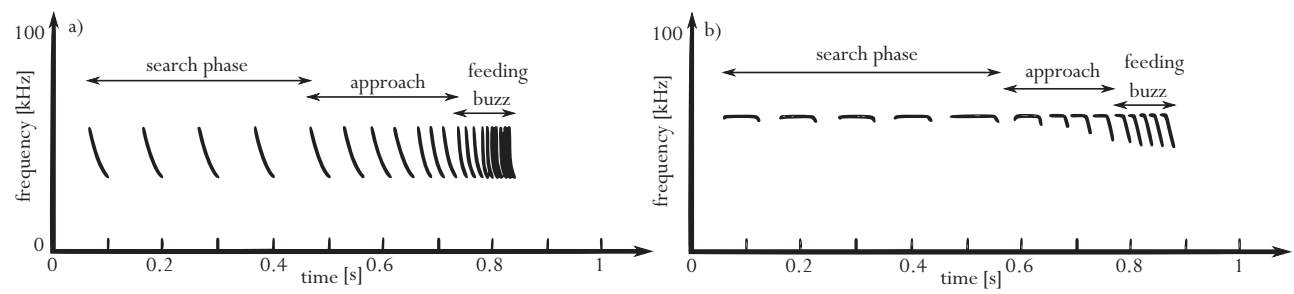

Fig. 3. Echolocation sequence of FM (a) and CF (b) bats emitted during prey pursuit. The pulse repetition rate increases when bats detect a prey reaching the maximum rate just before a catch.

\subsection{How bats detect faint echoes?}

The detection of echoes encounter the problems related to the echo strength and the interference of echoes of different individuals. Echolocation calls of most bats are very loud at the source but they are very quickly attenuated in the air, because most of the bats use ultrasound calls of high frequencies. So the returning echo is very weak. Moreover, bat's auditory system is exposed to extraneous sounds, e.g. sonar of other bats, which may interfere with bat's own echo processing. Bat overcome these problems by opening neural gate of short duration. The neural gate is triggered by the bat's own echolocation pulses, when they are emitted. This process briefly opens time windows enabling the bat's auditory system to receive and interpret bat's own echoes Roverud \& Grinnell (1985), Altringham (1999), Neuweiler (2000). The detection of weak pulses may be additionally enhanced by the process called forward masking. During forward masking hearing thresholds increase when acoustic stimuli are presented shortly after a sound of similar spectral composition, i.e. weak echoes returning shortly after the emission of their own comparatively intense sonar calls Siewert I. (2004).

\subsection{Determination of sonar target features}

The echo imagination of an object is affected by the structure of the echolocation pulse and the physical parameters of an object. FM and CF calls are adapted in a different way to intercept the objects. CF bats concentrate all or most of the energy into one frequency what carry the call further. CF signals are adapted to detect wing beats of fluttering insect in clutter environment, whereas FM pulses are well suited for three dimensional target localization, giving broadband 
echospectra. FM bats spread the energy over a broadband FM pulse with low frequency components. Some FM bats use also shallow FM pulses with low frequency CF tail. Some of them, e.g. noctule Nyctalus noctula, also alternate CF with FM pulses. This strategy is used by bats foraging in the open environments and helps bats to detect prey simply by maintaining the intensity of the pulse, and hence that of the echo, over greater distance Neuweiler (2000).

\subsubsection{Target location in 3-dimensional space}

FM bats discriminate an object's location in horizontal and vertical planes, the distance to the object and its relative velocity and direction of movement. For an analysis of the distance to a target an echolocating FM bat uses the time interval (delay) between their emitted sonar vocalization and a returning echo to obtain target distance information Simmons (1973). An FM bat appears to determine prey velocity primarily by measuring the changes in distance between it and its prey from one echo to the next.

The horizontal position of an object in space is determined by differences in the intensity and/or time of arrival of echoes at the two ears Fig. (4). Tragus plays probably an important role in the vertical positioning, since it is responsible for generating multiple reflections in the external ear as echoes travel to the eardrum Lawrence \& Simmons (1982). The reflections of sound waves from tragus and the wall of the pinna create interference patterns, which change according to the vertical direction of the sound Fig. (4). Therefore the vertical positioning may be resolved by analyzing secondary echoes which follow different paths through the inner ear and around the tragus, depending upon their direction of origin. The big brown bat Eptesicus fuscus can resolve vertical angles down to $3.5[\mathrm{deg}]$, and horizontal angles of $1.5[\mathrm{deg}]$. Bats that lack tragus (horseshoe bats and hipposiderids) move their pinnae in a pattern correlated with the emission of their echolocation calls: one ear moves upward and downward, the second ear moves forward and back. This pinnae movements play a role in sound localization a the vertical plane Neuweiler (2000).

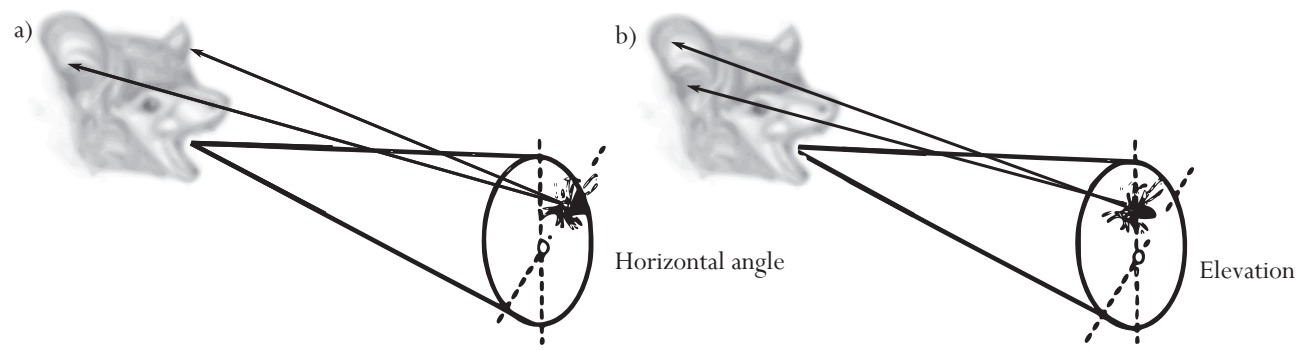

Fig. 4. The schematic imagination of the vertical and horizontal angles determination by an echolocating bat.

\subsubsection{Target range differences discrimination}

FM bats well resolve the discrimination of small differences in the distance to two objects, utilizing matched-filter and the envelope of autocorrelation function. The bat cross-correlates the emitted pulses with returning echo. The ACR describes the average period $T$ of the signal (the reciprocal of the fundamental frequency, which decreases with increasing frequency) and $t$, the width of the envelope at the half of its maximum. This is approximately the reciprocal of the signal's bandwidth. The introduction of FM higher harmonics decreases the height of the side peaks, reducing $t$. Bat neural system can determine unambiguously the timing (i.e. 
position) of the peak of the ACR. The narrower the central peak, and the lower the side peaks, the more acute the bat's target ranging. It is achieved with the FM pulses of large bandwidth (small $t$ ), high fundamental frequency (small $T$ ) and with additional harmonics (the side peaks are of low amplitude). The CF bat (constant frequency call with short, narrow bandwidth FM tail) can only distinguish between two objects if one is more than $12[\mathrm{~mm}]$ closer from the bat than the other. The FM bat can distinguish distance of $4[\mathrm{~mm}]$, which means that it is able to differentiate between echoes arriving as little as $20[\mu \mathrm{s}]$ apart Fig. (5) Simmons (1973).

Important role in target ranging plays the neural gate. It acts as a distance filter for bat's own sound. In Noctilio albiventris, Eptesicus fuscus and Rhinolophus rouxi the time window is open for around $30[\mathrm{~ms}]$, allowing the bats to target range over distances up to $5[\mathrm{~m}]$. The bat can ignore echoes from more distant objects Altringham (1999).

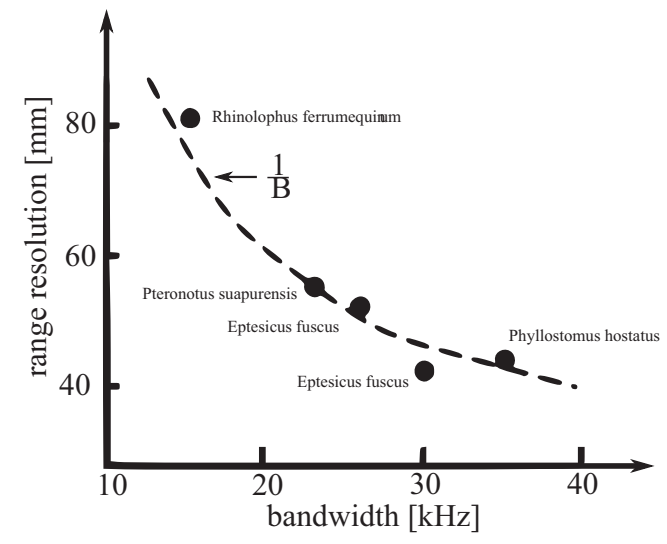

Fig. 5. Relationship between call bandwidth $(B)$ and target ranging accuracy in several species of bat (Rhinolophus and Pteronotus are known to used CF, the remaining species are FM bats) (from Altringham (1999) based on Simmons (1973))

\subsubsection{Determining object size}

Bats determine target size using amplitude modulation, i.e. the intensity of the echo. The bigger an object reflecting echolocation pulses, the smaller amplitude modulation. By small objects the amplitude of returning echo is decreasing. Different species of bats can detect and avoid wires with a diameter of $0.06-0.1[\mathrm{~mm}]$.

\subsubsection{Determining object structure}

Most of the objects perceived by bats is very complex and have many reflective surfaces that result in a chaotic acoustic image of the target without systematic spectral interference pattern. The echo perceived by a bat consists of its ultrasonic emission convolved with the acoustic impulse response (IR) of the ensonified object. The IR is the sum of reflections from the complex target. Its extraction implies great echolocation capabilities of bats. The experiment with Phyllostomus discolor (Phyllostomidae) showed that bats can classified echoes and evaluate IR roughness. Hence bats can evaluate complex texture, such as e.g. foliage type Grunwald et al. (2004). Thus the structure of more complex objects, ensonified by ultrasounds, can be probably detected with high precision by echolocating bats. This capability allow bats to recognize not only environment features but also find and classify prey or food source 
inaccessible for other (bat) species. This ability is especially important in FM bats, that forage in a clutter environment and detect insects against background or feed on nectar and pollen of flower. The precision in structure classification and insect detection in a clutter leads to niche partitioning in FM clutter forager bat species, because prey detection in clutter increases as the width of the FM call band broadens Siemers \& Schnitzler (2004)

Some of the flower-visiting bats (glossophagines from the family Phyllostomidae) can probably estimate the stage of flower inflorescense using echolocation. Mucuna holtoni, a bat-pollinated flower attracts bats by conspicuous echoes. The plant open new flowers successively from dusk to about midnight, so the bat must search for newly open flowers which serve with pollen. When a bud becomes mature, its vexillum (upper petal) is raised. The experiments showed that the vexillum indicate the presence of mature flowers to the bats. Based on this feature, exploded flowers can be distinguished from virgin flowers Helversen \& Helversen (1999). When the flowers of Mucona holtoni are exposed to artificial sounds (imitating echolocation calls of bats), they give different echoes (amplitude modulations) in bud and open flower. The raised vexillum strongly reflect sounds so the echo of the whole flower is strongly dominated by echo of the vexillum Helversen O. von (2003).

The best performance in determining target structure is achieved by FM bats. As opposed to CF calls, FM signals contain more information about spectral changes (modulation of the frequency components) and amplitude changes (modulation of the strength of the echo). The very broadband FM call $(100-20[\mathrm{kHz}])$ used by Megaderma lyra enables it to analyse textural depths in the range $0.9-4.2[\mathrm{~mm}]$. The narrowband FM tail to the call of greater horseshoe bats is limited to $1.1-1.5[\mathrm{~mm}]$ Altringham (1999). Experiments with Megaderma lyra showed that the bat can detect the difference in depth of only $0.2[\mathrm{~mm}]$ Schmidt (1992). The bat can do that not only using echolocation but also by passive listening to the virtual echoes.

Bats can perceived sound structure by analyzing the interference patterns of echos. Signals reflecting from the rough structure contains peaks and nulls at different frequencies, depending on the depth of the textural surface of the target. The interference between sound waves of identical frequency and amplitude causes addition or cancellation of the energy. The addition of the energy is maximal when the waves are in phase, whereas maximal cancellation occurs when the echo and emitted signal are $180[\mathrm{deg}]$ out of phase. Such cancellation within a spectrum is called a notch. A notch occurs when the distance between the high and low points on a target is $\lambda / 4$ or an uneven multiple of this relationship. The addition of the energy occurs when the differences in depth between the target surface is $\lambda / 2$ or even multiple thereof Fig. (6). The position of the peaks and nulls within the spectrum provides a measure of the surface texture of an object. The smoother target the higher the frequency of the maximal notch Neuweiler (2000).

\subsubsection{CF calls and prey detection in CF bats}

CF bats intercept the space and prey in a different way as FM bats. The CF pulses has poor range resolution, although they also need determine the range for interception or avoidance. The CF bats probably therefore introduce FM component to their signals. These bats detect moving prey using the Doppler shift (Doppler effect) of the constant frequency or by detecting the changes in echoes of a flying insect caused by its wing beats Schnitzler et al. (1983), Schnitzler (1987). The echo reflected from the fluttering insect contains momentary glint-like energy maxima. Such glints consists of amplitude and frequency modulations. Changes in wing profile causes variation in the intensity of the echo amplitude modulation. The wing movement towards or away from the direction of the bat broadens sound spectrum due to 


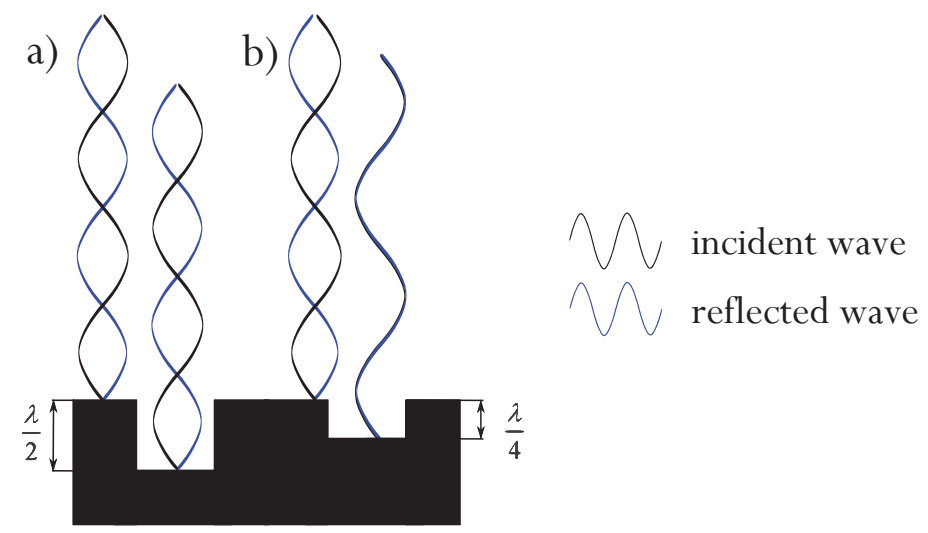

Fig. 6. The interference pattern of sounds reflecting from the object if rough structure.

Doppler effects. Echoes received by a bat approaching a stationary insect will be increased in frequency, and increased further if the insect is approaching the bat Schnitzler et al. (1983), Schnitzler (1987). Similarly, echospectra of fluttering insect oriented at three different angles demonstrates that the echo will be amplitude modulated within a pulse, and there will be also fluctuations in echoes from pulse to pulse Schnitzler et al. (1983). Insects of different size and from different species/families can therefore give different amplitude modulation, which may be an useful information for foraging bats.

The Doppler shift arises from the differences in velocity between bat and insect and the CF bats can compensate for this effect Schnitzler (1968). FM pulses are Doppler tolerant, so they are not affected significantly by either their own motion or that of the prey. CF bats compensate for Doppler shift induced by bat's flight speed by lowering the frequency of emitted pulses when flying. Doppler shift compensation causes that emitted pulse and returning echo differ in frequency, however the frequency of returning echo always falls within the range of the frequency emitted when resting, which is the frequency of best hearing ' $(\mathrm{BF})^{\prime}$. CF bats are very sensitive to a very narrow frequency band '(BF)', actually one frequency of CF signal, which is called auditory fovea (Neuweiler (2000), see the next chapter). Therefore the bat alters its emission frequency to keep an echo at a strictly defined frequency. The shift in emitted frequency tells the bat how fast it is moving, and in what direction, relative to the object. As a target moves and the echo frequency changes, the bat shifts its emitted frequency to keep the echo frequency constant Neuweiler (2000).

\subsection{Auditory adaptations to sound perception}

Bats auditory system is adapted to special tasks of the spatial orientation using sound (pulse and its echo) to perceive the environment, but it is built and operate in the similar manner as in other mammals, i.e. pinna with tragus, external ear, middle ear and inner ear, auditory nerve and auditory centers in brain Neuweiler (2000).

\subsubsection{Functional anatomy of the ear}

Echolocating bats have relatively large pinnae. The pinna acts as movable directional antenna. The directional hearing and sound picking by the pinna depends on the size and geometry of the pinna as well as the wavelength of sound. The pinna functions as a directional antenna when $k a>1.25$, that is, when the wavelength is smaller than $4-5$ times the radius of the 
pinna opening where $a$ - radius of the pinna opening. The larger the pinna, the better low frequencies will be transmitted to the eardrum. In Plecotus auritus, a bat with relatively big pinnae, the cut-off frequency is $6-8[\mathrm{kHz}]$. The pinna can not exert its directionality when the wavelength of sound exeeds the dimensions of the pinna. The higher the frequency, the more pronounced the directional characteristic of the pinna Neuweiler (2000).

The highly variable pinna form and size in different families of bats affect their acoustic properties. The pinna in most echolocating bats is rather small, because it is tuned to ultrasound frequencies with short wavelengths between 2 and $16 \mathrm{~mm}$. In contrast, in gleaning bats (taking prey from the surface) which listen to the low frequency rustling noise made by prey, the pinna is extraordinarly large. The greatest pinna amplification is in the range of $6-10[k \mathrm{~Hz}]$.

Unique to most echolocating bats is the tragus, cartilaginous projection at the base of the pinna. Tragus enhances discrimination of the elevation of the target Lawrence \& Simmons (1982). Bats that do not posses tragus have antitragus, a horizontal fold of the skin along the opening of the ear canal, and frequently move their pinnae.

Sound perceived by pinna passes to middle ear and causes vibration of eardrum (tympanic membrane), which is only $2-11[\mu \mathrm{m}]$ thick in echolocating bats. The vibrations are passed to the oval window along 3 ears ossicles: malleus, incus and stapes. Those media acts as filters, because of the vibration capabilities. The higher frequencies bats produce and perceive the thinner the eardrum is and smaller and lighter middle ear ossicles, because they vibrate more rapidly. Vibrations of the oval window are transmitted along the spiral canal of the cochlea. Cochlea is a bone with three canals spiral in parallel with the organ of Corti between them. It is highly complicated sound receiver and frequency analyzer, which categorizes the sound by frequency. Echolocating bats have $2.5-3.5$ complete turns, relative to the 1.75 of non-echolocating bats and primates Altringham (1999).

The inner canal of the cochlea is a tube, scala media, filled with fluid. It is covered by a second tube, which upper part is called scala vestibuli and the lower part scala tympani. Both of those tubes are separated from middle ear by membranes of the oval and round windows Fig. (7). The floor of the scala media is formed by the basilar membrane with sensory hair cells. Each hair cell posses bundle of stereocilia. The sterocilia tips are covered by the tectorial membrane. Vibration of the basilar membrane against the tectorial membrane causes shearing of the hair cell sterocilia and consequently, oscillations in the receptor potential that follow the rhythm of basilar membrane movement. This movement is caused by the momentary pressure differences between the scala vestibuli and scala tympani, which arises when the stapes moves and press against the oval window. The basilar membrane moves up and down at the same frequency as the stimulus. The oscillation progresses along the length of the basilar membrane, decreasing its speed. When the stimulus is a high frequency sound, the travelling wave moves only a short distance along the basilar membrane. The lower the frequency, the farther the traveling wave moves within the cochlea. Thus, high frequencies activate only the most basal hair cells, and lower frequencies activate the apical hair cells most strongly Fig. (7) Neuweiler (2000). Each hair cells is activated by specific frequency, its best frequency (BF). The frequency map of the basilar membrane varied between CF and FM bats. The CF bats have 'personal' constant frequency which show small variation in a resting specimen $( \pm 50$ $[\mathrm{Hz}]$, Schnitzler (1968)). Therefore there is a mechanical filter in cochlea tuned to very narrow frequency band of about several $\mathrm{kHz}$. The cochlea of $\mathrm{CF}$ bats has the expanded representation of a narrow frequency band of only $6[\mathrm{kHz}]$ around the individual's pure-tone echo frequency Fig. (8). The hair cells sensitive to that narrow band occupy the entire second turn of the 
cochlea, what correspond to one-fourth of the length of the basilar membrane. In FM bats this region contains the representation of about one octave and the frequency progression of the hair cells id approximately logarithmic Vater et al. (1985), Neuweiler (2000). This very narrow filter in CF bats is called auditory fovea. The auditory fovea of each individual bat is precisely tuned to its own emitted frequency Vater et al. (1985). The corresponding fitter exists in the in consecutive stages of sound processing in the brain (see next subchapter).

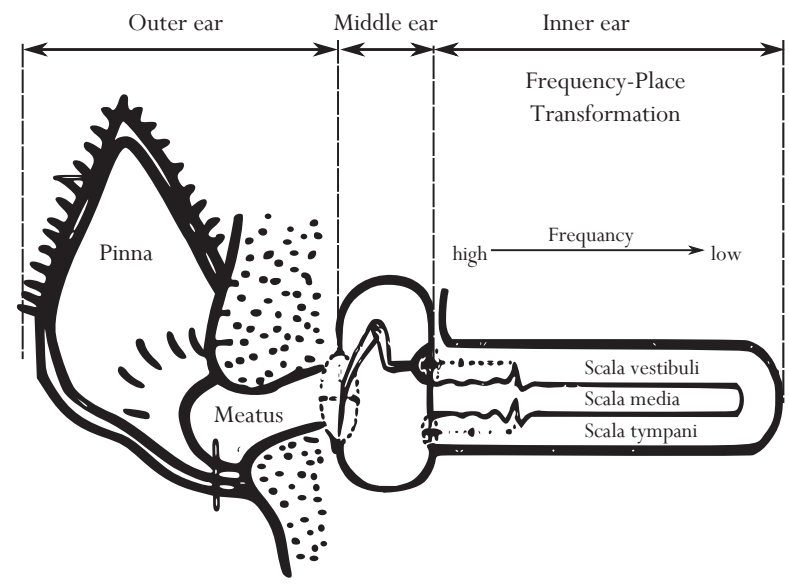

Fig. 7. The schematic plan of the mammalian ear and sound wave flow from the pinna to the inner ear (adapted from Neuweiler (2000)).

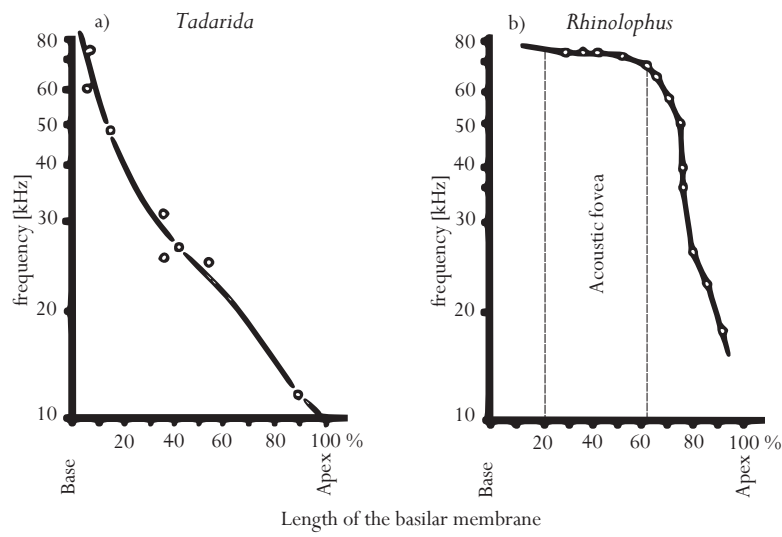

Fig. 8. Frequency representation on the basilar membrane in the inner ear of FM (Tadarida brasiliensis) and CF bats (Rhinolophus rouxi). CF bat show specific auditory fovea (adapted from Vater et al. (1985) and Neuweiler (2000).

\subsubsection{Auditory pathways in the central nervous system}

The central auditory system of bats consists of the same nuclei as in other mammals, however some of these nuclei are relatively larger than they are in other mammals. The impulses from the hair cells in the inner ear are transfer to the auditory nerve fibers, which further transmit 
the impulse to the midbrain auditory centre, called inferior colliculus. In echolocating bats the inferior colliculus is large. It gather all pathways together and transmits information to the medial geniculate body of the thalamus. From thalamus the information is proceeds to auditory cortex in forebrain where the final processing of the sound is happened and sound is translated into images of the environment Neuweiler (2000). The output of the bank of

FM bat

Eptesicus fuscus

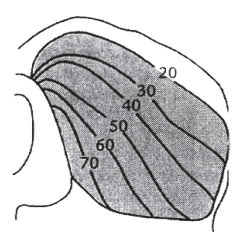

CF bat

Hipposideros speoris

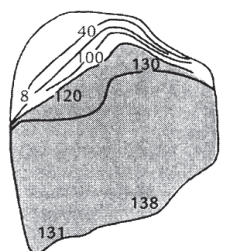

Fig. 9. Tonotopic frequency representation in the inferior colliculus in FM bat (Eptesicus fuscus) and CF bat (Hipposideros speoris) (from Ostwald (1984))

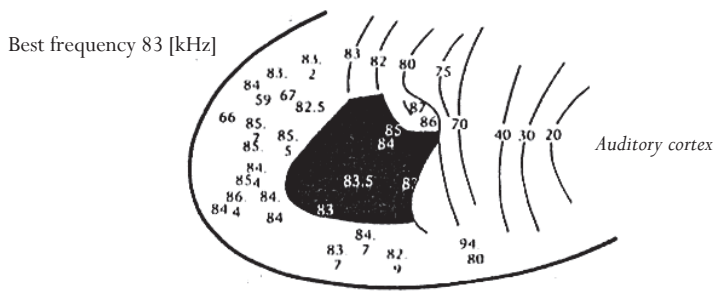

Fig. 10. Tonotopic frequency representation in the auditory cortex of CF bat (Rhinolophus ferrumequinum) with narrow frequency band of auditory fovea (from Neuweiler (2000).
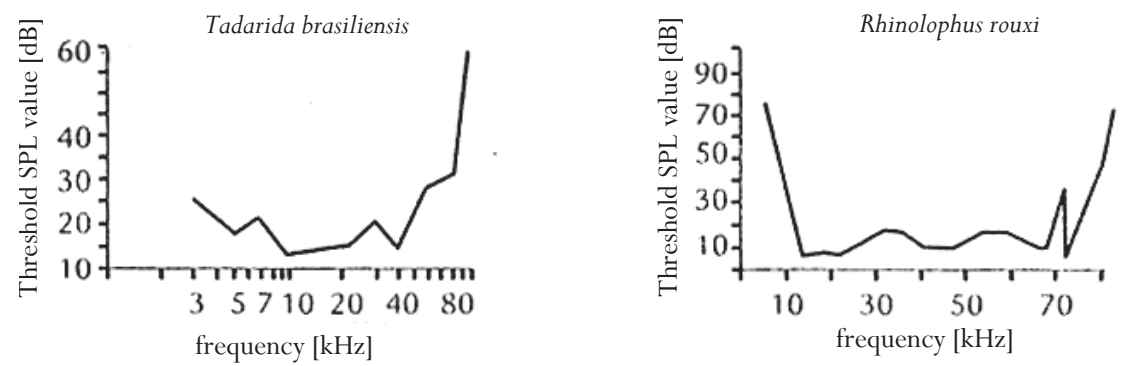

Fig. 11. Audiograms of FM (Tadarida brasiliensis) and CF bats (Rhinolophus rouxi) obtained from the measurements of the neurons in the inferior colliculus. CF bat shows fine tuning to a narrow frequency band (at about $73[\mathrm{kHz}]$ ) of the auditory fovea (adapted from Vater et al. (1985), Neuweiler (2000).

spectral filters in the inner ear is organized into a tonotopic (cochlear) map with a laminar arrangement of neurons of the inferior colliculus. The cells within a given lamina all have a similar best frequency Fig. (9). Thus, each lamina is responsible for processing information 
from one specific frequency band. In FM bat frequency bands are distributed less or more evenly, whereas in CF bat, the lamina responsible for one narrow frequency band is relatively large. The auditory cortex of $\mathrm{CF}$ bat has adequate an overrepresentation of the neurons sensitive to the frequencies of only $1.5[\mathrm{kHz}]$ band Fig. (10). These narrow filters correspond to the auditory fovea and is seen in the auditory tuning curves of CF bats, as a region of very low threshold for the frequencies of CF portion of the echolocation pulse Schuller \& Pollak (1979) Fig. (11). In FM bats the neurons are almost always either broadly tuned or only fairly sharp Neuweiler (2000) Fig. (11).

\section{Ultrasound waves in gas medium}

The nature of a gas medium, which is determined by its parameters, is extremely ultrasound unfriendly in relation to ultarasound waves generation transmission and detection. The main parameter of the gas medium is sound velocity $c$. It varies depending on such parameters as static pressure and temperature of gas. A theoretical dependence between sound velocity and gas properties is given by Eq. (1)

$$
c=\sqrt{\frac{\kappa(T) \cdot p_{0} \cdot T}{\rho_{0} \cdot T_{0}}}
$$

where $\kappa(T)$ - ratio of specific heats $c_{p} / c_{v^{\prime}}, p_{0}$ - static pressure in normal conditions, $\rho_{0}$ - gas density in normal conditions, $T_{0}-293 \mathrm{~K}$. The main problems in case of an air coupled sonar system based on TOF are temperature and pressure changes, which have to be compensated using external sensors or reference channels. The second important parameter which is critical in sonar systems is sound attenuation in a given medium. In gases sound attenuation is described by clasicall Stokes-Kirchhoff Eq. (2)

$$
\alpha_{c l}=\alpha_{\eta}+\alpha_{\sigma}=\frac{\omega^{2}}{2 \rho c^{3}}\left[\frac{4}{3} \eta^{\prime}+\sigma\left(\frac{1}{c_{v}}-\frac{1}{c_{p}}\right)\right]
$$

where $\omega$ - circular frequency, $\eta^{\prime}$ - shear viscosity, $c_{v}$ - specific heat at a constant volume, $c_{p}$ - specific heat at a constant pressure, $\sigma$ - heat conductivity. The most significant information contained in Eq. (2) is that the attenuation factor is proportional to the square of circular frequency $\alpha \approx \omega^{2}$. As a result of the above the range of air operating sonar system is significantly limited to a few centimeters for frequencies above $100[\mathrm{kHz}]$. Additionally, echolocating bats use band limited signals from the range of $20-200[\mathrm{kHz}]$. This varies between species, but in some cases also depends on hunting strategy and the type of surrounding obstacles. A precise expression of attenuation in air Eq. (3) was formulated by Evans et al. (1972).

$$
\alpha=\frac{2 \pi^{2} f^{2}}{\kappa c_{0} p_{0}}\left(\frac{4}{3} \eta^{\prime}+\frac{\kappa-1}{c_{p}} \sigma+\kappa a_{d} D_{12} \rho\right)
$$

The model of sound absorbtion in air takes into account both the classical processes (viscous losses, heat conduction) and relaxation. The two new variables $a_{d}$ and $D_{12}$ stand for molecular diffusion constant, and diffusion coefficient for the mixture of oxygen and nitrogen repectievly. The relation between attenuation and frequency for different humidity is shown in Fig. (12)

Considering the simplest case of an acoustic TRX (Transmitting - Receiving) system made of a coaxially situated single transmitter and receiver shown in Fig. (13) it is possible to define 

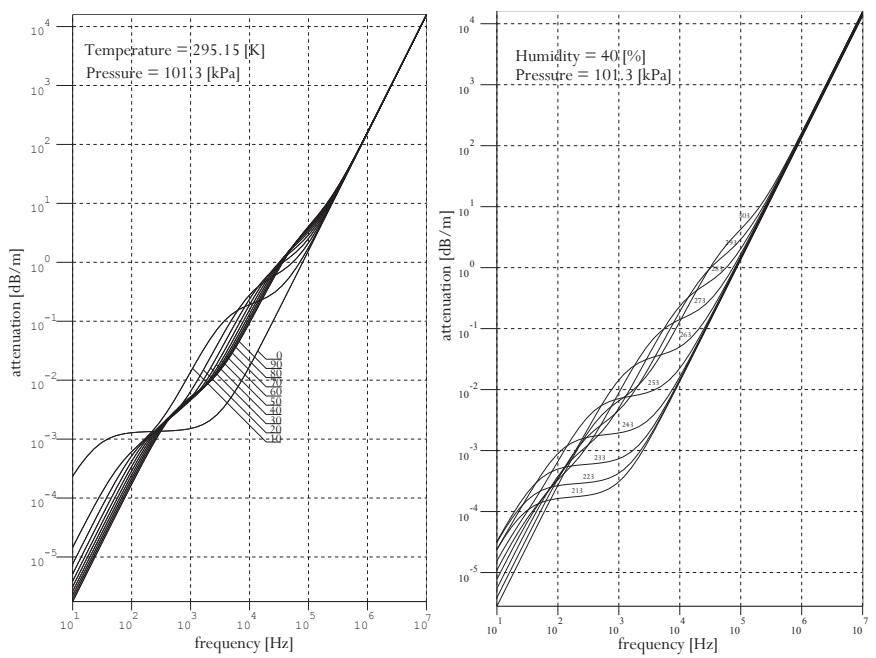

Fig. 12. Attenuation coefficient as a function of frequency with various parameters: humidity in a range of $0-90[\%]$ with 10 percent resolution (left), temperature in a range of $213-303$ $[K]$ with $10[K]$ resolution (right). According to Evans et al. (1972) and Bass et al. (1995).

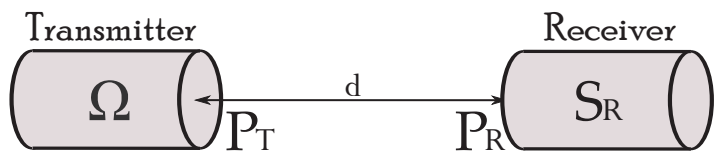

Fig. 13. An illustration of a simple acoustic link

acoustic link range equation (4), widely described by Gudra \& Opielinski (2006).

$$
\frac{P_{T}}{P_{R}}=\frac{4 \pi d^{2} e^{2 \alpha d}}{\Omega \cdot S_{R}}
$$

The equation defines a relation between both transmitted and received acoustic power as a function of distance $d$, directivity of the transmitter $\Omega=\pi^{2} f^{2} D_{T}^{2} / 2 c^{2}$ for $\left.\left.\pi f D_{T} / 2 c\right\rangle\right\rangle 1$, receiver's surface $S_{R}$ and attenuation factor $\alpha$.

The third important limitation, especially important in air coupled sonar systems, is acoustic impedance mismatch. According to transmission line theory the energy transmission coefficient equals to Eq. (5)

$$
T=2 \frac{\sqrt{Z_{1} Z_{2}}}{Z_{1}+Z_{2}}
$$

In most ultrasonic applications the acoustic impedance of the transmitter or receiver is a few order higher than the impedance of the medium. As a result of this phenomena most acoustic energy is reflected from impedance discontinuity. Some methods of reducing the impedance mismatch will be described in section (4).

Target strength TS is another very important parameter in the context of object detection using a sonar system. This parameter characterises the reflecting properties of an object and is defined by Eq. 6)

$$
T S=10 \log _{10}\left(\frac{I_{r}}{I_{I}}\right)
$$


where $I_{r}$ and $I_{t}$ indicate intensities of reflected and incident waves respectively Because of the fact that target strength depends on objects' geometry and their reflecting capabilities it can be calculated for simple solids like sphere, cylinder etc. More information about target strength measurements for different objects can be found in the papers Gudra et al. (2010) and Norman \& Jones (2000). To evaluate the performance of an active sonar operating in air we include target strength TS Eq. (7)

$$
S L-2 T L+2 T S-N L+D I+P T>R T
$$

where: $S L$ - source level, $T L$ - transmission losses, $N L$ - noise level, DI - directivity index, PG processing gain, $R T$ - reception threshold.

\section{Air coupled ultrasound transducers}

Since air coupled acoustic signal is used in passive and active sonar systems, ultrasound transducers have to be applied in these solutions. Due to problems related to ultrasound signals transmission described in section (3) signal transmitters and receivers have to guarantee some critical parameters in order to achieve eligible results. Efficiency $S_{n}$ and sensitivity $S_{0}$ are very basic parameters of acoustic transmitters/receivers Due to the transducer dimensions comparable with an acoustic wave length the ultrasonic sensors are usually highly directional. The directional parameters of transducers are described by directivity coefficient $\Omega$ given by Eq. (8)

$$
\Omega=\frac{2}{\int_{0}^{\pi} K^{2}(\theta) \sin (\theta) d \theta}
$$

The directivity characteristic for a circular piston vibrating in an infinite baffle is described by Eq. (9)

$$
K(\theta)=\frac{2 J_{1}\left(\frac{\pi f D_{T} \cdot \sin (\theta)}{c}\right)}{\frac{\pi f D_{T} \cdot \sin (\theta)}{c}}
$$

where $D_{T}$ - piston diameter, $J_{1}$ - Bessel function of first order. The problem of simplifying the Eq. (9) is widely described by Gudra \& Opielinski (2004). In practice for the directivity coefficient calculations of ultrasonic transducers working in the air it can be assumed that:

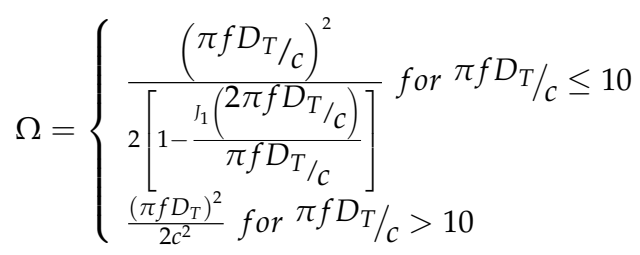

There is a variety of transducers that are used to generate or receive ultrasounds in a gas medium. Some selected properties of the transducers are presented in a table (1)

A dynamic transducer may be used to produce low frequency ultrasound. The basic limitation of cut-off frequency is high impedance of vibrating coil and decrease of effective radiating surface of the loudspeaker membrane. An electro-mechano-acoustical model of dynamic loudspeaker is shown in Fig. (14). The parameters of analog circuit and it analysis are widely 


\begin{tabular}{|c|c|c|c|}
\hline Type & Frequency range & Transmitter & Receiver \\
\hline Dynamic & broadband & Yes & No \\
\hline Condenser & broadband & Yes & Yes \\
\hline Piezocermic & resonant & Yes & Yes \\
\hline PVDF & resonant/boadband & Yes & Yes \\
\hline EMFi & broadband & Yes & Yes \\
\hline Electret & broadband & Yes & Yes \\
\hline
\end{tabular}

Table 1. Comparison of ultrasound transducers' basic properties.

described in a book Borwick (2001). The most important conclusion is that the SPL produced by this type of transducer decreases 12 [dB/oct] because of voice coil impedance $L_{c}$ and air mass load $M_{m d}$.
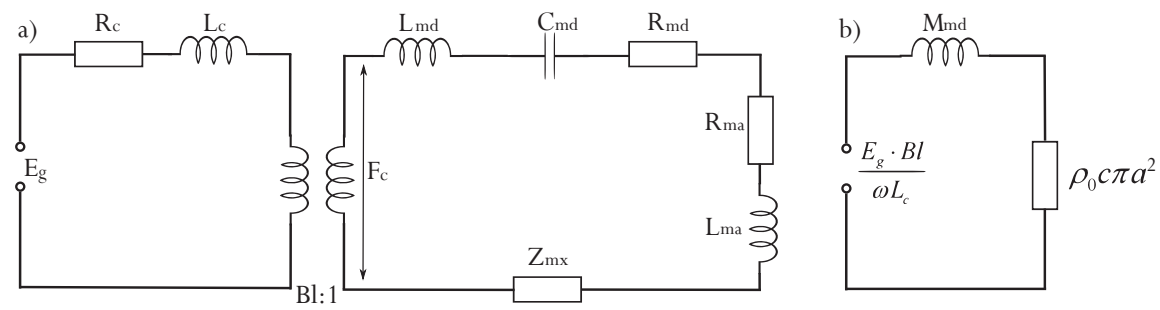

Fig. 14. Dynamic transmitter model a) in a full range of frequencies, b) at very high frequencies (above 2-nd resonance).

The next group of transducers associated with ultrasound wave generation are condenser microphones and transmitters. Most of ultrasound microphones are based on condenser receiver principle. The common known limitation of such a device is its small membrane surface in comparison to the wave length, which causes low sensitivity. The microphones are also very susceptible to humidity and dirt. Condenser transmitters are not as popular as the receivers due to low effectiveness, but in a range of frequencies above $50[\mathrm{kHz}]$ condenser construction is, next to EMFi film, the only broad band alternative. The most common material in ultrasound transducers technology is piezoceramics. This is due to the resonant character of generated waves and its highest effectiveness/sensitivity. Since Langevine developed a sandwich type transducer in 1927, it has been the most known type of transducer, next the one that uses piezoceramic rings vibrating in radial symmetrical mode. The main advantage of sandwich type transducer coupled with vibrating plate is its high effectiveness and ability to work continuously and generate high acoustic power. Additionally, a surface of vibrating plate improves energy transmission coefficient Eq. (5). A typical construction of a sandwich transducer is shown in Fig. (15).

Sandwich transducer elements' dimensions are described by Eq. (11).

$$
\operatorname{tg}\left(\frac{2 \pi d f_{0}}{c_{p}}\right) \cdot \operatorname{tg}\left(\frac{2 \pi b f_{0}}{c_{b}}\right)=\frac{Z_{p}}{Z_{b}}
$$

where:

$Z_{p}$ - impedance of piezoceramics material,

$Z_{b}$ - impedance of loading mass material,

$c_{b}$ - speed of sound in loading mass material,

$c_{b}$ - speed of sound in piezoceramics material, 


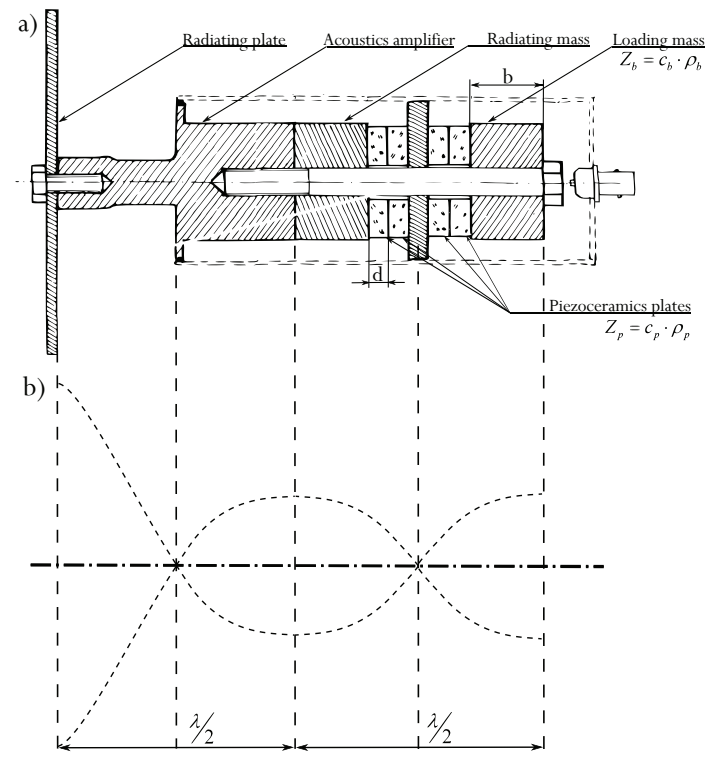

Fig. 15. Sandwich transducer a) structure of a transducer with vibrating plate, b) acoustic velocity distribution.

$d$ - thickness of piezoceramic plate,

$b$ - thickness of loading mass,

$f_{0}$ - resonance frequency.

The length requirements for acoustic velocity transformer is presented in Fig. (15 b) and equals $\lambda / 2$. Radiating mass length has to fulfill requirements $\lambda / 4$. The maximum radiating acoustic power can be achieved by applying stepped plate, which vibrates flexurally in its axial mode. The major problem of the presented construction is the high mechanical $Q$-factor of the plate and mechanical part of the transducer and electrical $Q$-factor of piezoceramics.

Radial and thicness vibration of piezoceramic disc are commonly used as a source of acoustic wave in many sensors. An piezoelectric disc and its electrical model are shown in Fig. (16). Radial $u_{r}$ and thickness $u_{z}$ displacement of radiating surfaces are given by Eq. (12).

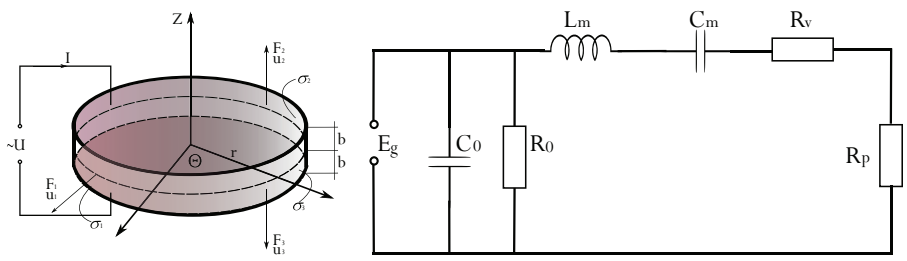

Fig. 16. Coordinate system, force distribution, dimensions of piezoelectric disc and its electrical equivalent circuit at resonance frequency.

$$
\begin{gathered}
u_{r}=-\frac{u_{1}}{j \omega J_{1}\left(k_{1} a\right)} J_{1}\left(k_{1} r\right) \\
u_{z}=\frac{1}{j \omega}\left[-\frac{\left(u_{2}+u_{3}\right)}{2 \sin \left(k_{3} b\right)} \sin \left(k_{3} z\right)+\frac{\left(u_{3}-u_{2}\right)}{2 \cos \left(k_{3} b\right)} \cos \left(k_{3} z\right)\right]
\end{gathered}
$$


where:

$$
k_{1}=\frac{\omega}{c_{1}}, c_{1}=\sqrt{\frac{c_{11}^{D}}{\rho}}, k_{2}=\frac{\omega}{c_{3}}, c_{3}=\sqrt{\frac{c_{33}^{D}}{\rho}},
$$

The thickness vibrations of disc with matching layer generate quasi-plane wave while the radial ones are the source of cylindrical wave. To produce a wave with reduced near field and side lobes it is possible to use radial vibrations of the piezoceramic disc to actuate the transducer mechanical enclosure and radiate the wave in $z$ direction.

Maximum displacement across $x$ direction in thickness vibration mode occures at $r=0$. . Radial vibration displacement reaches its maximum at $r=a$ across $r$ direction. Normalised displacement across both directions as a function of radius is shown in Fig. (17)

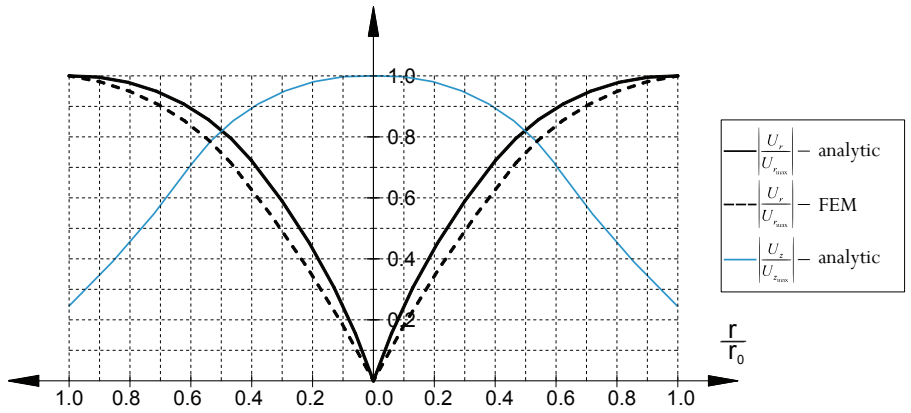

Fig. 17. Distribution of radial and thickness components of displacements of piezoelectric disk calculated using analytical method and FEM (according to Gudra (2008))

The acoustic energy in air operating active sonar systems is transmitted from transducer to a gas medium. The impedance of the piezoceramics PZT equals $Z_{p}=29.6 \cdot 10^{6}$ while the air acoustic impedance equals $Z_{\text {air }}=427$. One of the methods to reduce the adverse energy transmission coefficient Eq. (5) is to use impedance matching layer as it is shown in Fig. (18). Using one-quarter of the wave length matching layer made of material the acoustic impedance of which is $Z_{p}=1.12 \cdot 10^{5}$ guarantees maximum energy transmission. The matching layer material is usually made of composite porous materials. Another way to improve impedance matching is applying multilayered matching structure, where $Z_{k}<<$ $Z_{k+1}$. Sound attenuation in particular layers is a limitation of this method.

The problem of impedance mismatch is less significant for EMFi (Electromechanical Film) based transducers. The physical and acoustics parameters of foil are presented in papers by Paajanen et al. (2000) and Paajanen et al. (2001). The most important advantages of EMFi film is high value of longitudinal piezoelectric constant $d_{33}$, which varies in the range of $130-450[p C / N]$ and low value of acoustic impedance, which equals $2.6 \times 10^{4} \mathrm{~kg} /\left(\mathrm{m}^{2} \cdot \mathrm{s}\right)$ EMFi film could be used as a broadband transmitter/receiver and can produce bats-like echolocation pulses. This attribute was used in some robotic applications described in Jiménez et al. (2008), Steckel et al. (2008) and Schillebeeckx et al. (2008). Since the bats echolocation mechanism is the most sophisticated biosonar, engeeniers have attempted to use it as a pattern and inspiration in their echolocation devices. Target position estimation is made on the basis of echo signal processing. Target position is specified by range $d$, azimuth $\phi$ and elevation $\theta$ angles. 


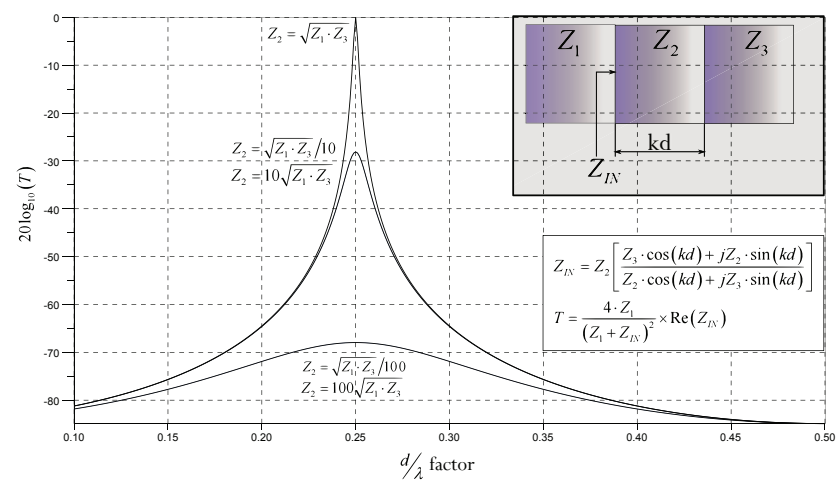

Fig. 18. Impedance matching using one layer and transmission line theory according to Lynnworth (1965).

\section{Signal processing in sonar systems}

Nowadays, ultrasound signal processing in air-coupled sonar systems is dominated by digital technology. Digital signal processing techniques give engineers a whole spectrum of possibilities and enables them to adapt the equipment to specified solutions. On the other side, every ultrasound device consists of an analog signal processing block. A generalised ultrasound localisation system is shown in Fig. (19). Each echolocation system can be configured using the proposed generalisation. The extreme simplification of presented system could be realised as one TRX transducer with analog filter, an amplifier and a fullwave rectifier in the receiving circuit plus an analog switching circuit and a step-in transformer in the transmitting circuit. The ADC block is based on an analog comparator and DAC block consists of an analog switching circuit driver. Everything is controlled by microcontroller which drives DAC and measures the time it takes comparator signal to reach the unit. Much more interesting echo analyzing could be applied using full analog to digital conversion and DSP algorithms. One of the most popular examples in ultrasound ranging system is based on autocorrelation of digitised $x(k)$ echo signal Eq. (14).

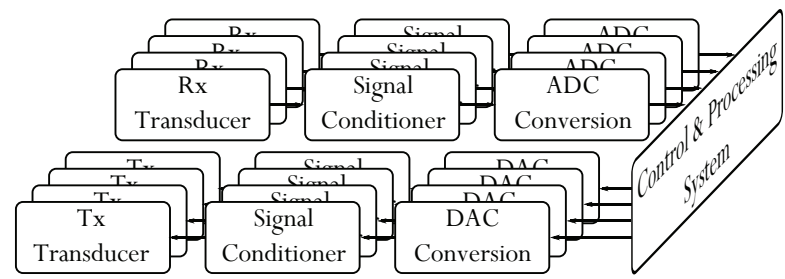

Fig. 19. Generalised block scheme of air operating echolocation system.

$$
y(n)=\sum_{k=0}^{N-1} x(k) x(k+n)
$$

A signal processing simulation of an echo from a two different targets using CF (Constant Frequency) Eq. (15) and LFM (Linear Frequency Modulation) Eq. (16) signals is shown in Fig. 
(20).

$$
\begin{gathered}
s_{C F}(n)=A \cdot \sin \left(2 \pi \frac{f_{0}}{f_{s}} n\right) \\
s_{L F M}(n)=A \cdot \sin \left[2 \pi\left(f_{0}+\frac{m}{2} \cdot \frac{n}{f_{s}}\right) \cdot \frac{n}{f_{s}}\right]
\end{gathered}
$$

Analysis of the results obtained using different signals proves that the modulation of the

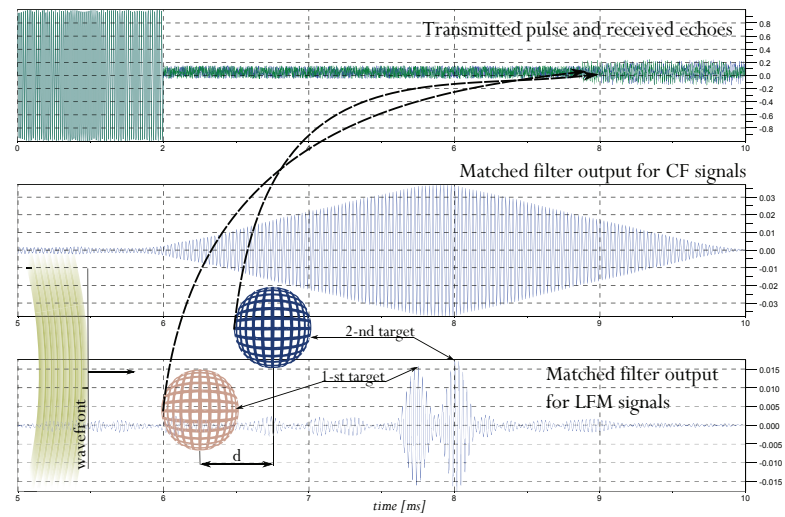

Fig. 20. Simulation of target ranging capabilities using pulse compression technique.

Echolocation system performance - $f_{s}-400[\mathrm{kHz}], f_{0}-40[\mathrm{kHz}]$ (for CF wave) and $f_{0}-40$ $[\mathrm{kHz}]$ and $m-10$ (for LFM wave). Signal to noise ratio is set to $6[\mathrm{~dB}]$ and the target separation distance $d$ equals $8.5[\mathrm{~cm}]$.

transmitted signal ensures better target discrimination and range resolution. This phenomena is used by bats as it shown in section (2). Bats also reproduce direction of arrival DOA of the echo signals. The ILD Interaural Level Difference and ITD Interaural Time Difference are a source of information about DOA. In air coupled ultrasound signal processing systems the ITD parameter is commonly used due to attenuation changes across parameters described in section (3) and different target strengths for different objects. Some interesting information about ILD processing by bats and modeling of the bats' hearing mechanism using VLSI elements could be found in the paper by Shi \& Horiuchi (2007). Interaural time differences follows from the spatial grid of receiving sensors and the sound speed in a gas medium. Since the assumption is that wavefront returning echo signal is plane, we can evaluate horizontal and elevation angle using sensor arrays and beamforming algorithms. Additionally, there are methods allowing beam steering in a particular direction. This enables "looking" using ultrasounds in a selected direction. Using multiple receivers (an array of transducers in different configurations see. Fig. (21) it is possible to modify the directional characteristics of the array. This technique is commonly used in underwater hydrolocation systems and radars. There were some attempts of developing an ultrasonic air-operating beamformers for echolocation systems described in papers by Strakowski et al. (2006), Kleeman \& Kuc (1995), Medina \& Wykes (2001), Harput \& Bozkurt (2008) and Webb \& Wykes (1996), Wykes et al. (1993). Most of the solutions cited above are based on a linear array and the signal processing method used is time-delay beamforming see Johnson \& Dudgeon (1993.). Additionally, the authors developed an ultrasonic array for bats inspired echolocation study presented in Fig. (22) and described in Herman \& Stach (2011). In the proposed solution time-delay algorithm 


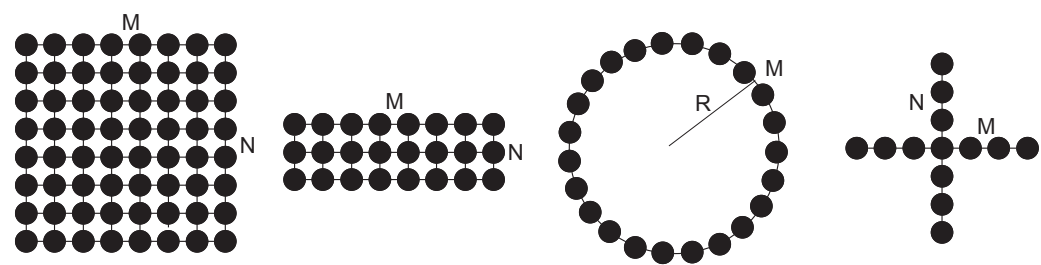

Fig. 21. Some regular grids of sensor arrays, based on Johnson \& Dudgeon (1993.)

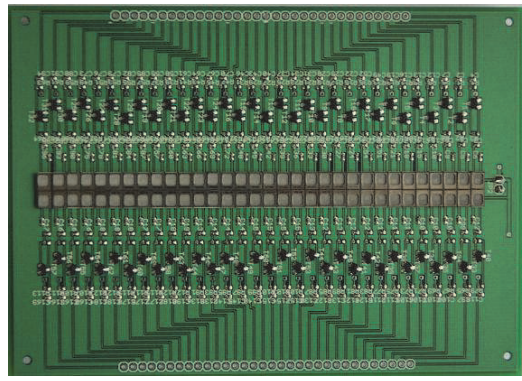

Fig. 22. A MEMS based linear array developed by authors. Horizontal intersensor spacing is equal to $3.8[\mathrm{~mm}]$ and array size is $32 \times 2$.

was used for a $32 \times 2$ MEMS sensor array. Due to the fact that signal from each microphone is digitized with sampling frequency $f_{s}$, the minimal phase shift equals sampling interval. When using digital beamforming algorithms it is critical to delay a beam by a number of samples that corresponds to a given beam deflection angle. To ensure proper angular resolution, the sampling frequency is given by modified Eq. (17):

$$
f_{s}=\frac{c}{d} \cdot \frac{180}{\pi \cdot \Phi_{\min }}
$$

where $c$ - velocity of sound, $d$ - intersensor spacing in linear array, $\Phi_{\min }$ - minimum phase shift angle. To improve horizontal angle estimation performance, the time series was upsampled using interpolation algorithm for each channel. The minimum $\Phi_{\min }$ could be obtained assuming that it equals half of the parabolic width $P W$ (at 0 steering angle) of the linear array given by Eq. (18):

$$
P W=\frac{4 \cdot \sqrt{3}}{k \cdot d \cdot \sqrt{M^{2}-1}}
$$

where: $M$ - number of sensors. Considering the fact that pulse compression method combined with bats-like downsweept chirps, it is possible to process array signals using pathways shown in Fig. (23): The proposed solution was simulated using similar methodology as it is shown in Fig. ( (20), where the difference is that the acoustic echo signal is processed by a linear array of 32 MEMS sensors. The results of the simulation are presented in the Fig. (24). Unfortunately, increasing number of channels and sampling frequency takes results in the system not being able to operate in a real time. The system processing speed may be increased by paralleling channel information processing, reducing the number of sensors, applying so called redundant-array solution by Johnson \& Dudgeon (1993.) or combining differences at respective receivers with information determined from evaluation directional 


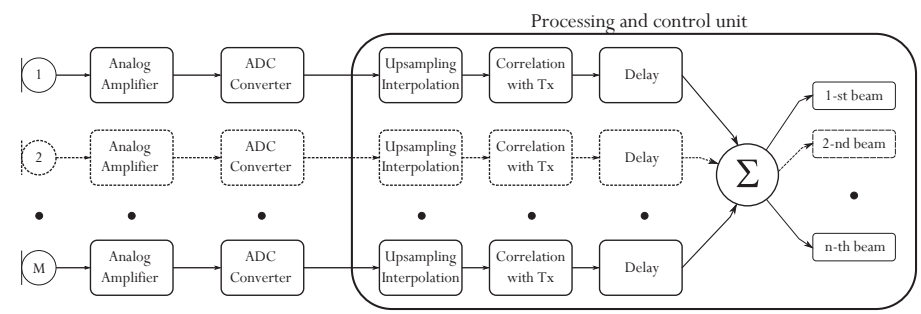

Fig. 23. Digital interpolation delay-sum beamforming scheme based on correlation of sensor data with transmitted signal Tx.
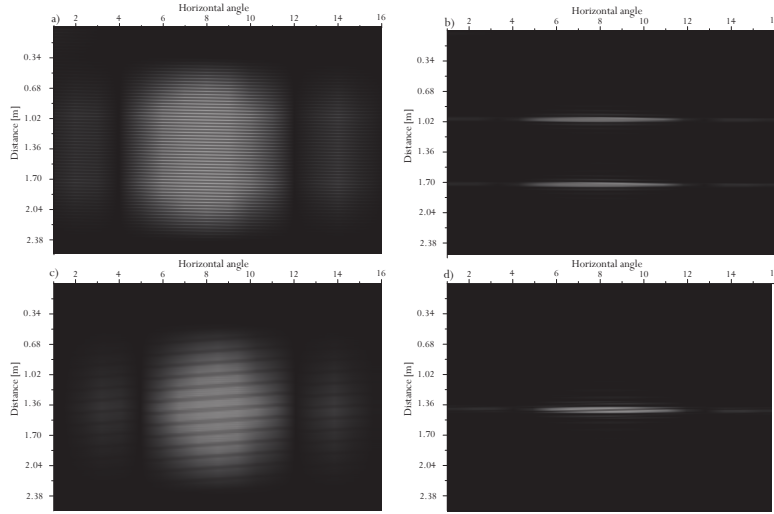

Fig. 24. A simulation of a target position estimation: a) multiple target with usage of CF signal, b) multiple target with usage of LFM signal, c) single target with usage of CF signal, d) single target with usage of LFM signal.

characteristics of the ultra sound transducers described in Georg Kaniak (2010). Due to the fact that processing speed of present-day signal technology is not increasing as rapidly as a few years before, we can observe that signal processing methods are migrating to multi-threaded calculations on multiprocessor platforms. An excellent illustration of the situation are the recent news in sonar technology samples Nilsen \& Hafizovic (2009), Coiras et al. (2009), where parallel computing of sensor data are being preformed on GPU targets.

\section{Conclusions}

The chapter describes selected matters associated with a variety of science fields such as: acoustics, electronics, signal processing and biology or more precisely chiropterology. It presents, in a concise manner, the properties of air as a gas milieu in the context of using it as a medium transmitting information encoded in ultrasonic waves. The performed analyses suggest that from the point of view of ultrasound technology air is a very unfavourable medium. This is evidenced by characteristics of gases and the methods of ultrasonic wave generation and reception. On the other side, this medium is used by bats, which can reproduce an image of the surrounding space using ultrasounds. Although current technology is far behind bats in the field of information processing, researchers are relentlessly trying to catch up and judging by the number of scientific publications interest in bats' echolocation is still high. The selected issues related to the bats' biology and presented in section 2 prove that bats 
can reproduce images of its surrounding with resolution exceeding the physical limitations arising from wave length. It is even possible to formulate a hypothesis stating that a bat is from a technological viewpoint "an intelligent multisensor", which not only processes acoustic information but also environmental conditions (temperature, humidity, flight speed). Some of the bat specific echolocation methods are applied in ultrasonic technology. These include simple distance (angle) meters based on processing information related to time period between signal transmission and echo reception but also advanced beamforming systems. The very structure of the signal used by bats is, however, a much more interesting subject. It appears that better space imaging is possible when using modulated signals, which, as was found, compensate Doppler effect and increase both range and angular resolution. As a direct application of this echolocation strategy, the authors present a solution based on the use of pulse compression method in digital beamforming. It was shown, by means of simulation, that such a system is characterised by a higher range resolution and is capable of more accurate discrimination of targets located at the same angle. As a next step in verifying simulation results, the developed solution will be tested on a platform specifically constructed for that purpose. The primary limitation of pulse compression method is the assumption that acoustic waves with frequency modulation will be generated. With present-day advancement of ultrasonic technology, the possibilities of broadband signal generation are very limited. Even if such a signal can be generated, its SPL level is significantly lower than in case of widely used and well developed resonance transducers. Since space imaging methods and models if reality prepared for air are often a modified transposition of the models used in hydrolocation, it is possible to use solutions and development directions from this field in the area of object localisation in air. Due to required resources and processor capacity it is likely that multibeam systems will turn to distributed computing on multiprocessor platforms. As mentioned in the work, one of possible applications, which has already been the subject of research of several scientific groups, is to construct a device that would help visually handicapped people to ambulate Abdul et al. (2008), Borenstein \& Ulrich (1997), Mihajlik et al. (2001), Shoval et al. (1994), Ifukabe et al. (1991). Considering the detail of the problem presented in this chapter and using bat signals and DSP algorithms utilised on an efficient computing platform, construction of such a device seems possible but will require an effort of an interdisciplinary research team.

\section{References}

Abdul, A. I., Sajee, M., Mazlina, M. \& Nazleeni, S. (2008). Blind echolocation using ultrasonic sensors, Information Technology, 2008 4: 1-7.

Altes, R. (1995). Signal processing for target recognition in biosonar, Neural Networks 8: 1275-1295.

Altringham, J. (1999). Bats. Biology and Behaviour, Oxford University Press Inc.

Au, W. W. L. (2004). Echolocation in Bats and Dolphins, The University of Chicago Press, Chicago and London, chapter A comparison of the sonar capabilities of bats and dolphins, pp. 12-27.

Balcombe, J. P. \& Fenton, M. B. (1988). Eavesdropping by bats: The influence of echolocation call design and foraging strategy, Ethology 79(2): 158-166.

Barclay, R. M. R. (1982). Interindividual use of echolocation calls: Eavesdropping by bats, Behavioral Ecology and Sociobiology 10: 271-275. 
Bass, H. E., Sutherland, L. C., Zuckerwar, A. J., Blackstock, D. T. \& Hester, D. M. (1995). Atmospheric absorption of sound: Further developments, The Journal of the Acoustical Society of America 97(1): 680-683.

Borenstein, J. \& Ulrich, I. (1997). The guidecane - a computerized travel aid for the active guidance of blind pedestrians, IEEE Int. Conf. on Robotics and Automation, pp. 1283-1288.

Borwick, J. (2001). Loudspeaker and Headphone Handbook, Focal Press.

Carmena, J. \& Hallam, J. (2004a). Narrowband target tracking using a biomimetic sonarhead, Robotics and autonomous systems 46(4): 247-259.

Carmena, J. \& Hallam, J. (2004b). The use of doppler in sonar-based mobile robot navigation: inspirations from biology, Information Sciences 161(1-2): 71-94.

Chou, T. N. \& Wykes, C. (1997). An integrated vision/ultrasonic sensor for 3d target recognition and measurement, Proc. Sixth International Conference on Image Processing and Its Applications, Vol. 1, pp. 189-193.

Coiras, E., Ramirez-Montesinos, A. \& Groen, J. (2009). Gpu-based simulation of side-looking sonar images, OCEANS 2009 - EUROPE, pp. 1 -6.

Dechmann, D. K., Heucke, S. L., Giuggioli, L., Safi, K., Voigt, C. C. \& Wikelski, M. (2009). Experimental evidence for group hunting via eavesdropping in echolocating bats, Proceedings of the Royal Society B: Biological Sciences 276 B: 2721-2728.

Ealo, J., Seco, F. \& Jimenez, A. (2008). Broadband emfi-based transducers for ultrasonic air applications, Ultrasonics, Ferroelectrics and Frequency Control, IEEE Transactions on 55(4): $919-929$.

Evans, L. B., Bass, H. E. \& Sutherland, L. C. (1972). Atmospheric absorption of sound: Theoretical predictions, The Journal of the Acoustical Society of America 51(5B): 1565-1575.

Georg Kaniak, H. F. S. (2010). Angle determination, scene analysis, ultrasound, Key Engineering Materials ( 437: 258-262.

Griffin, D. (1958). Listening in the dark, New Haven: Yale University Press.

Grunwald, J.-E., Schörnich, S. \& Wiegrebe, L. (2004). Classification of natural textures in echolocation, Proceedings of the National Academy of Sciences of the United States of America 101(15): 5670-5674.

Gudra, T. (2008). Ultrasounds in gas media: generation, transmission, aplications, Archives of Acousics 33(4): 581-592.

Gudra, T. \& Opielinski, K. (2004). Ultrasonic transducers working in the air with the continuous wave within the $50-500 \mathrm{khz}$ frequency range, ULTRASONICS 42(1-9): 453-458.

Gudra, T. \& Opielinski, K. J. (2002). Influence of acoustic impedance of multilayer acoustic systems on the transfer function of ultrasonic airborne transducers, Ultrasonics 40(1-8): $457-463$.

Gudra, T. \& Opielinski, K. J. (2006). The range equation of the ultrasonic link in gas media, ULTRASONICS 44(Suppl. 1): E1423-E1428.

Gudra, T., Opielinski, K. J. \& Jankowski, J. (2010). Estimation of the variation in target strength of objects in the air, Physics Procedia 3(1): 209 - 215. International Congress on Ultrasonics, Santiago de Chile.

Harput, S. \& Bozkurt, A. (2008). Ultrasonic phased array device for acoustic imaging in air, Sensors Journal, IEEE 8(11): 1755 -1762. 
Hartley, D. J. \& Suthers, R. A. (1987). The sound emission pattern and the acoustical role of the noseleaf in the echolocating bat, carollia perspicillata, The Journal of the Acoustical Society of America 82(6): 1892-1900.

Helversen, D. V. \& Helversen, O. V. (1999). Acoustic guide in bat-pollinated flower, Nature 759-760: 759-760.

Helversen O. von, W. Y. (2003). Bat ecology, University of Chicago Press. Chicago and London, chapter Glossophaginae bats and their flowers: costs and benefits for plants and pollinators, pp. 346-397.

Henson JR, O. W. (1965). The activity and function of the middle ear muscles in echolocating bats, J Physiol 180: 871-887.

Herman, K. \& Stach, L. (2011). Analysis and elaboration of an air-coupled ultra- sound wideband sensor array, Acta Physica Polonica A In Press.

Holderied, M. W., Baker, C. J., Vespe, M. \& Jones, G. (2008). Understanding signal design during the pursuit of aerial insects by echolocating bats: tools and applications, Integrative and Comparative Biology 48(1): 74-84.

Ifukabe, T., Sasaki, T. \& Peng, C. (1991). A blind mobility aid modeled after echolocation of bats, IEEE Transactions on Bio-Medical Engineering 38(5): 461-465.

Jiménez, A., Álvaro Hernández, Urena, J., Pérez, M. C., Álvarez, F. J., Marziani, C. D., García, J. J. \& Villadangos, J. M. (2008). Emfi-based ultrasonic transducer for robotics applications, Sensors and Actuators A: Physical 148(1): 342 - 349.

Johnson, D. \& Dudgeon, D. (1993.). Array Signal Processing, Prentice-Hall, Englewood Cliffs.

Jones, G. (1999). Scaling of echolocation call parameters in bats, J Exp Biol 202(23): 3359-3367.

Jones, G. (2008). Sensory ecology: echolocation calls are used for communication., Current Biology 18: R34-R3.

Kamata, E., Inoue, S., Zheng, M., Kashimori, Y. \& Kambara, T. (2004). A neural mechanism for detecting the distance of a selected target by modulating the fm sweep rate of biosonar in echolocation of bat, Biosystems 76(1-3): 55 - 64 .

Kay, L. (1964). An ultrasonic sensing probe as a mobility aid for the blind, Ultrasonics 2(2): 53 -59 .

Kleeman, L. \& Kuc, R. (1995). Mobile robot sonar for target localization and classification, International Journal of Robotics Research 14: 295-318.

Lawrence, B. \& Simmons, J. (1982). Echolocation in bats: the external ear and perception of the vertical positions of targets, Science 218(4571): 481-483.

Lynnworth, L. (1965). Ultrasonic impedance matching from solids to gases, Sonics and Ultrasonics, IEEE Transactions on 12(2): $37-48$.

Masters, W. M. \& Harley, H. E. (2004). Echolocation in Bats and Dolphins, The University of Chicago Press, Chicago and London., chapter Performance and Cognition in Echolocating Mammals, pp. 249-259.

Medina, L. \& Wykes, C. (2001). Multiple target 3d location airborne ultrasonic system, Ultrasonics 39(1): 19-25.

Mihajlik, P., Guttermuth, M., Seres, K. \& Tatai, P. (2001). Dsp-based ultrasonic navigation aid for the blind, Procceedings of the 18-th IEEE Instrumentation and measurement technology conference, Vol. 1-3, pp. 1535-1540.

Moss, C. \& Sinha, S. (2003). Neurobiology of echolocation in bats, CURRENT OPINION IN NEUROBIOLOGY 13(6): 751-758.

Neuweiler, G. (2000). The biology of bats, Oxford University Press Inc. Oxford. 
Nilsen, C.-I. \& Hafizovic, I. (2009). Digital beamforming using a gpu, Acoustics, Speech and Signal Processing, 2009. ICASSP 2009. IEEE International Conference on, pp. 609 -612.

Norman, A. P. \& Jones, G. (2000). Size, peripheral auditory tuning and target strength in noctuid moths, Physiological Entomology 25(4): 346-353.

Ostwald, J. (1984). Tonotopical organization and pure tone response characteristics of single units in the auditory cortex of the greater horseshoe bat, Journal of Comparative Physiology A: Neuroethology, Sensory, Neural, and Behavioral Physiology 155: 821-834.

Paajanen, M., Lekkala, J. \& Kirjavainen, K. (2000). Electromechanical film (emfi) - a new multipurpose electret material, Sensors and Actuators A: Physical 84(1-2): 95 - 102.

Paajanen, M., Lekkala, J. \& Valimaki, H. (2001). Electromechanical modeling and properties of the electret film emfi, Dielectrics and Electrical Insulation, IEEE Transactions on 8(4): 629 $-636$.

Peremans, H. \& Van Campenhout, J. (1993). Tri-aural perception on a mobile robot, Proc. IEEE International Conference on Robotics and Automation, pp. 265-270.

Peremans, H., Walker, A. \& Hallam, J. C. T. (1998). 3d object localisation with a binaural sonarhead, inspirations from biology, Proc. IEEE International Conference on Robotics and Automation, Vol. 4, pp. 2795-2800.

Roverud, R. C. \& Grinnell, A. D. (1985). Echolocation sound features processed to provide distance information in the $\mathrm{cf} / \mathrm{fm}$ bat noctilio albiventris: evidence for a gated time window utilizing both $\mathrm{cf}$ and fm components, Journal of Comparative Physiology A: Neuroethology, Sensory, Neural, and Behavioral Physiology 156: 457-469.

Schillebeeckx, F., De Mey, F. \& Peremans, H. (2008). Bio-inspired sonar antennae: Enhancing directivity patterns for localization, Proc. 2nd IEEE RAS \& EMBS International Conference on Biomedical Robotics and Biomechatronics BioRob 2008, pp. 366-371.

Schmidt, S. (1992). Perception of structured phantom targets in the echolocating bat, megaderma lyra, The Journal of the Acoustical Society of America 91(4): 2203-2223.

Schnitzler, H., D., M., R., K. \& K, H. (1983). The acoustic image of fluttering insects in echolocating bats, Neuroethology and Behavioural Physiology 3: 236-250.

Schnitzler, H. U. (1968). Die ultraschall-ortungslaute der hufeisen-fledermaeuse (chiroptera: Rhinolophidae) in verschiedene orientierungssituationen, Journal of Comparative Physiology A: Neuroethology, Sensory, Neural, and Behavioral Physiology 57: 376-408.

Schnitzler, H. U. (1987). Recent advances in the study of bats, Cambridge University Press, Cambridge., chapter Echoes of fluttering insects: information for echolocating bats, pp. 226-243.

Schuller, G. \& Pollak, G. (1979). Disproportionate frequency representation in the inferior colliculus of doppler-compensating greater horseshoe bats: Evidence for an acoustic fovea, Journal of Comparative Physiology A: Neuroethology, Sensory, Neural, and Behavioral Physiology 132: 47-54.

Shi, R. Z. \& Horiuchi, T. K. (2007). A neuromorphic vlsi model of bat interaural level difference processing for azimuthal echolocation, IEEE Transactions on circuits and systems 54(1): 74-88.

Shoval, S., Borenstein, J. \& Koren, Y. (1994). Mobile robot obstacle avoidance in a computerized travel aid for the blind, IEEE International Conference on Robotics and Automation, pp. 8-13.

Siemers, B. M. \& Schnitzler, H.-U. (2004). Echolocation signals reflect niche differentiation in five sympatric congeneric bat species, Nature 429(6992): 657-661. 
Siewert I., Schillinger T., S. S. (2004). Echolocation in Bats and Dolphins, The University of Chicago Press, Chicago and London, chapter Forward masking and the consequences on echo perception in the gleaning bat, Megaderma lyra, pp. 266-268.

Simmons, J. A. (1973). The resolution of target range by echolocating bats, The Journal of the Acoustical Society of America 54(1): 157-173.

Steckel, J., Reijniers, J., Boen, A. \& Peremans, H. (2008). Biomimetic target localisation using an emfi based array, Proc. 17th IEEE International Symposium on the Applications of Ferroelectrics, Vol. 3, pp. 1-2.

Strakowski, M. R., Kosmowski, B. B., Kowalik, R. \& Wierzba, P. (2006). An ultrasonic obstacle detector based on phase beamforming principles, IEEE Sensor Journal 6(1): 179-186.

Surlykke, A. \& Kalko, E. K. V. (2008). Echolocating bats cry out loud to detect their prey, PLoS ONE 3(4): e2036.

Vater, M., Feng, A. S. \& Betz, M. (1985). An hrp-study of the frequency-place map of the horseshoe bat cochlea: Morphological correlates of the sharp tuning to a narrow frequency band, Journal of Comparative Physiology A: Neuroethology, Sensory, Neural, and Behavioral Physiology 157: 671-686.

Webb, P. \& Wykes, C. (1996). High-resolution beam forming for ultrasonic arrays, Robotics and Automation, IEEE 12(1): 138-146.

Wykes, C., Nagi, F. \& Webb, P. (1993). Ultrasound imaging in air, Proc. International Conference on Acoustic Sensing and Imaging, pp. 77-81. 


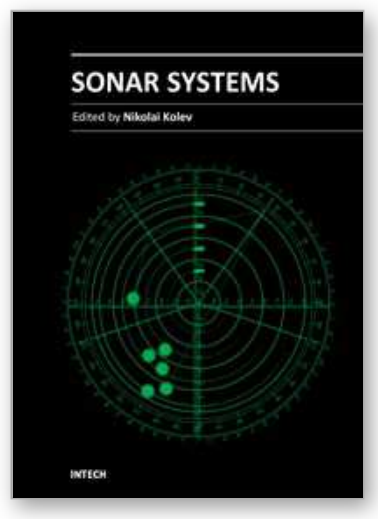

\author{
Sonar Systems \\ Edited by Prof. Nikolai Kolev
}

ISBN 978-953-307-345-3

Hard cover, 322 pages

Publisher InTech

Published online 12, September, 2011

Published in print edition September, 2011

The book is an edited collection of research articles covering the current state of sonar systems, the signal processing methods and their applications prepared by experts in the field. The first section is dedicated to the theory and applications of innovative synthetic aperture, interferometric, multistatic sonars and modeling and simulation. Special section in the book is dedicated to sonar signal processing methods covering: passive sonar array beamforming, direction of arrival estimation, signal detection and classification using DEMON and LOFAR principles, adaptive matched field signal processing. The image processing techniques include: image denoising, detection and classification of artificial mine like objects and application of hidden Markov model and artificial neural networks for signal classification. The biology applications include the analysis of biosonar capabilities and underwater sound influence on human hearing. The marine science applications include fish species target strength modeling, identification and discrimination from bottom scattering and pelagic biomass neural network estimation methods. Marine geology has place in the book with geomorphological parameters estimation from side scan sonar images. The book will be interesting not only for specialists in the area but also for readers as a guide in sonar systems principles of operation, signal processing methods and marine applications.

\title{
How to reference
}

In order to correctly reference this scholarly work, feel free to copy and paste the following:

Tadeusz Gudra, Joanna Furmankiewicz and Krzysztof Herman (2011). Bats Sonar Calls and its Application in Sonar Systems, Sonar Systems, Prof. Nikolai Kolev (Ed.), ISBN: 978-953-307-345-3, InTech, Available from: http://www.intechopen.com/books/sonar-systems/bats-sonar-calls-and-its-application-in-sonar-systems

\section{INTECH}

open science | open minds

\section{InTech Europe}

University Campus STeP Ri

Slavka Krautzeka 83/A

51000 Rijeka, Croatia

Phone: +385 (51) 770447

Fax: +385 (51) 686166

www.intechopen.com

\section{InTech China}

Unit 405, Office Block, Hotel Equatorial Shanghai

No.65, Yan An Road (West), Shanghai, 200040, China

中国上海市延安西路65号上海国际贵都大饭店办公楼 405 单元

Phone: $+86-21-62489820$

Fax: $+86-21-62489821$ 
(C) 2011 The Author(s). Licensee IntechOpen. This chapter is distributed under the terms of the Creative Commons Attribution-NonCommercialShareAlike-3.0 License, which permits use, distribution and reproduction for non-commercial purposes, provided the original is properly cited and derivative works building on this content are distributed under the same license. 